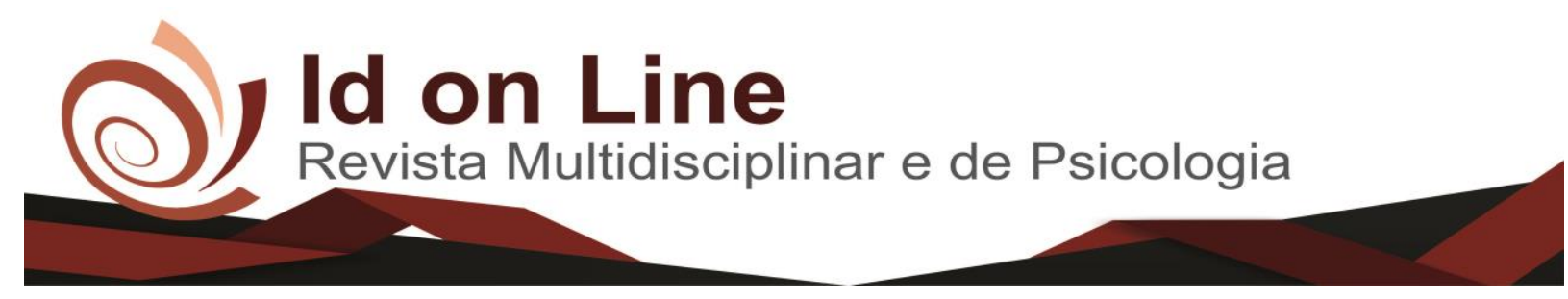

Artigo

\title{
Avaliação da influência de transtornos mentais sobre a pressão arterial em idosos cadastrado em um projeto
}

\author{
Gilmar Santos Silva ${ }^{1}$; Valquíria Santos Gomes ${ }^{2}$; Maria Aparecida Silva Santos Macêdo ${ }^{3}$
} Stênio Fernando Pimentel Duarte ${ }^{4}$;

\begin{abstract}
Resumo: A Hipertensão Arterial Sistêmica (HAS) é um estado clinico causado por múltiplos fatores, que tem como características graus aumentados e mantidos de pressão arteriais sendo variáveis psicológicas exercem uma grande influencia no seu aparecimento. Objetivo: analisar a influência de algumas variáveis psicológicas nos níveis de pressão arterial no individuo idoso Métodos: trata-se de estudo do tipo analítico com delineamento transversal e abordagem quantitativa, as coletas de dados foram realizadas nos meses de agosto e setembro de 2016 e teve como objeto de estudo 238 indivíduos em um município do interior da Bahia. Resultados: Quanto ao cruzamento entre os dados de transtornos mentais e hipertensão arterial: 11 não hipertensos apresentavam distúrbios psicológicos enquanto 200 apresentavam hipertensão arterial concomitantemente á distúrbios mentais. Conclusão: O presente estudo evidenciou uma relação direta entre hipertensão arterial e distúrbios psicológicos.
\end{abstract}

Palavras-chave: hipertensão arterial sistêmicas-has; depressão; ansiedade; estresse.

\section{Evaluation of the influence of mental disorders about arterial pressure in elderly registered in a project}

\begin{abstract}
Systemic arterial hypertension (SAH) is a clinical state caused by multiple factors, which has as characteristics increased degrees and sustained arterial pressure and psychological variables have a great influence on their appearance Objective: to analyze the influence of some psychological variables on blood pressure levels in the elderly. Method: This is an analytical study with a cross-sectional design and a quantitative approach. Data collection was carried out in August and September 2016, and 238 individuals were studied in a municipality in the interior of Bahia. Results: Regarding the cross-referencing of mental disorders and hypertension: 11 non-hypertensive patients presented psychological disorders while 200 presented arterial hypertension concomitantly with mental disorders. Conclusion: The present study evidenced a direct relationship between arterial hypertension and psychological disorders.
\end{abstract}

Keywords: systemic hypertension-sah; depression; anxiety; stress.

\footnotetext{
${ }^{1}$ Graduando em Enfermagem pela Faculdade Independente do Nordeste (FAINOR). E-mail:gilmarss2005@hotmail.com Vitória da Conquista, Bahia, Brasil.

${ }^{2}$ Enfermeira Esp, graduada pela Universidade do Sudoeste da Bahia(UESB). E-mail: valquiriasgomes@gmail.com - Vitória da Conquista, Bahia, Brasil.

${ }^{3}$ Graduanda de Enfermagem pela faculdade Independente do Nordeste (FAINOR). E-mail: lyasoyer@ hotmail.com. Vitória da Conquista, Bahia, Brasil.

4 Biólogo. Professor da Faculdade Independente do Nordeste (FAINOR). Doutor em Ciências (Clínica e Experimental). E-mail: sfernando@fainor.com.br Vitória da Conquista, Bahia, Brasil.
} gilmarss2005@hotmail.com. 


\section{Introdução}

A Hipertensão Arterial Sistêmica (HAS) é um estado clinico causado por múltiplos fatores, que tem como características graus aumentados e mantidos de pressão arterial (BRASIL, 2013). É uma patologia considerada atualmente como um dos mais importantes problemas de saúde de ordem pública, tanto na esfera brasileira como na esfera global, sendo ela a causa principal de morte ou de forma indireta age concomitantemente para que esta ocorra em milhões de pessoas (PEREZ, 2015). Em torno de 32,5\% (36 milhões) de indivíduos brasileiros é acometido pela hipertensão arterial e entre a população idosa há um amento desse percentual que chega á 50\%, sendo responsável por 339.672 dos quais $(29,8 \%)$ das mortes por doenças cardiovasculares (MALACHIAS et al., 2016).

Existe varias classificações do grau de hipertensão arterial sistêmica-HAS essa classificação é feita a partir da medida da pressão sistólica e diastólica é considerada ótima se os níveis estiverem entre: Normotensos se a pressão sistólica estiver $<120 \mathrm{mmHg}$ e a diastólica $<80 \mathrm{mmHg}$ é considerada normal os níveis de pressão sistólica $<130 \mathrm{mmHg}$ e diastólica < $85 \mathrm{mmHg}$, Limítrofe pressão sistólica entre 130 a 139 mmHg e diastólica entre 85 a $89 \mathrm{mmHg}$ e considerada como Hipertensão estágio 1 se a pressão sistólica estiver entre 140 a $159 \mathrm{mmHg}$ e a diastólica estiver entre 90 a $99 \mathrm{mmHg}$, Hipertensão estágio 2 se a pressão sistólica estiver entre 160 a 179 mmHg e a diastólica 100 a 109 mmHg, Hipertensão estágio 3 se a pressão sistólica estiver $\geq 180 \mathrm{mmHg}$ e a diastólica estiver $\geq 110 \mathrm{mmHg}$ (BRASIL, 2013).

A depressão é considerada um transtorno do humor e tem como sintomas característicos a apatia a dificuldade de exercer as atividades diárias, insônia ou hipersonia e desejo de auto-extermínio entre outros (APA, 2013). Já o estresse é uma resposta do organismo frente a fatores estressores (CAVALCANTI JUNIOR; SOUZA, 2016).

Uma hipótese é que a depressão possa estar ligada a resposta imune, e a sínteses de catecolaminas onde esse mecanismo age deficitariamente o que contribui etiologicamente para o individuo desenvolver tanto depressão como estresse (Rabelo et al., 2017). Atualmente fala se muito sobre a influência dos substâncias químicas produzidas pelos neurônio tendo em destaque os neurotransmissores dopamina e serotonina (MOREIRA; GARCIA; CAVAZZA, 2917).

Tendo em vista o grande número de idosos que apresentam concomitantemente algum distúrbio psicológico e Hipertensão Arterial Sistêmica (HAS), este presente estudo teve com 
objetivo analisar a influência de algumas variáveis psicológicas nos níveis de pressão arterial no individuo idoso, para tal foi eleito os distúrbios mentais: ansiedade, depressão e estresse.

\section{Metodologia}

Trata-se de um subprojeto do projeto principal intitulado Perfil Epidemiológico da Obesidade e os fatores associados, um estudo do tipo analítico com delineamento transversal e abordagem quantitativa, as coletas de dados foram realizadas nos meses de agosto e setembro de 2016 e teve como objeto de estudo 238 indivíduos em um município do interior da Bahia.

Para atingir o objetivo foram utilizados os seguintes instrumentos de coleta de dados: Questionário de estresse de Lipp "O questionário de estresse foi organizado por LIPP e teve sua validação em 1994, teve sua reformulação e padronização e aprovação em 2000, permitindo assim fazer o diagnostico de estresse ou sua ausência em indivíduos adultos. Este questionário é Composto por três fases a primeira referem a sintomas físicos e mentais das ultimas 24 horas, o segundo fase refere a sintomas de experimentado na semana anterior e ultima fase refere a sintomas apresentado no mês anterior” (ROSSETTI et al, 2008, p. 113).

Questionário de depressão de Beck "O inventario de depressão de Beck é um questionário onde o individuo sinaliza os sintomas da depressão e ele permite qualificar o grau de depressão ou sua ausência” (GOMES-OLIVEIRA et al., 2012, p. 390).

Questionário de ansiedade de Beck "Os sintomas de ansiedade foram analisados pelo Inventário de Ansiedade de Beck (BAI), que é composto por 21 itens onde o sujeito precisa assinalar, em uma grandeza de quatro pontos, o grau dos sintomas que ele refere, onde as classificações variam entre grau mínimo grau leve, grau moderado ou grave" (SILVA et al., 2016, p. 230).

Questionário socioeconômico adaptado - Universidade Estadual de Londrina (UEL) "Para classificar a classe social em que o individuo estudado pertence foi aplicado o questionário simplificado a partir do questionário socioeconômico elaborado pela Universidade Estadual de Londrina-PR. Que composto por perguntas referende a renda familiar" (FINATTI; ALVES; SILVEIRA, 2007, p. 1). 
Quanto a pressão Arterial foi aferida a partir deum aparelho digital de marca G-TECH usada à técnica de aferição preconizada pelo Ministério da Saúde (BRASIL, 2013).

Vale salientar que todos os participantes do estudo assinaram livremente o Termo de Consentimento Livre e Esclarecido. Em seguida, foram agendados os encontrados para coleta dos dados, que aconteceu de maneira individualizada e respeitando a rotina de trabalho do grupo. A análise de dados foi obtida com auxílio do Programa Statistical Package for the Social Sciences SPSS® 22.0, considerando um nível de significância de $\mathrm{p}<0,05$. O presente esta inserido no Núcleo de Extensão, Pesquisa e Estudo de Doenças Crônicas, que visa mapear o perfil da obesidade de um município do interior da Bahia estudo seguiu os preceitos éticos e legais e teve todo de desenvolvimento desse trabalho foi conduzido de acordo com a Resolução 466/12 e submetido à aprovação do Comitê de Ética em Pesquisa da Faculdade Independente do Nordeste (FAINOR), que tem como parecer de aprovação nº 1.670.007.

\section{Resultados e Discussão}

Tabela 1 - Transtornos e hipertensão arterial

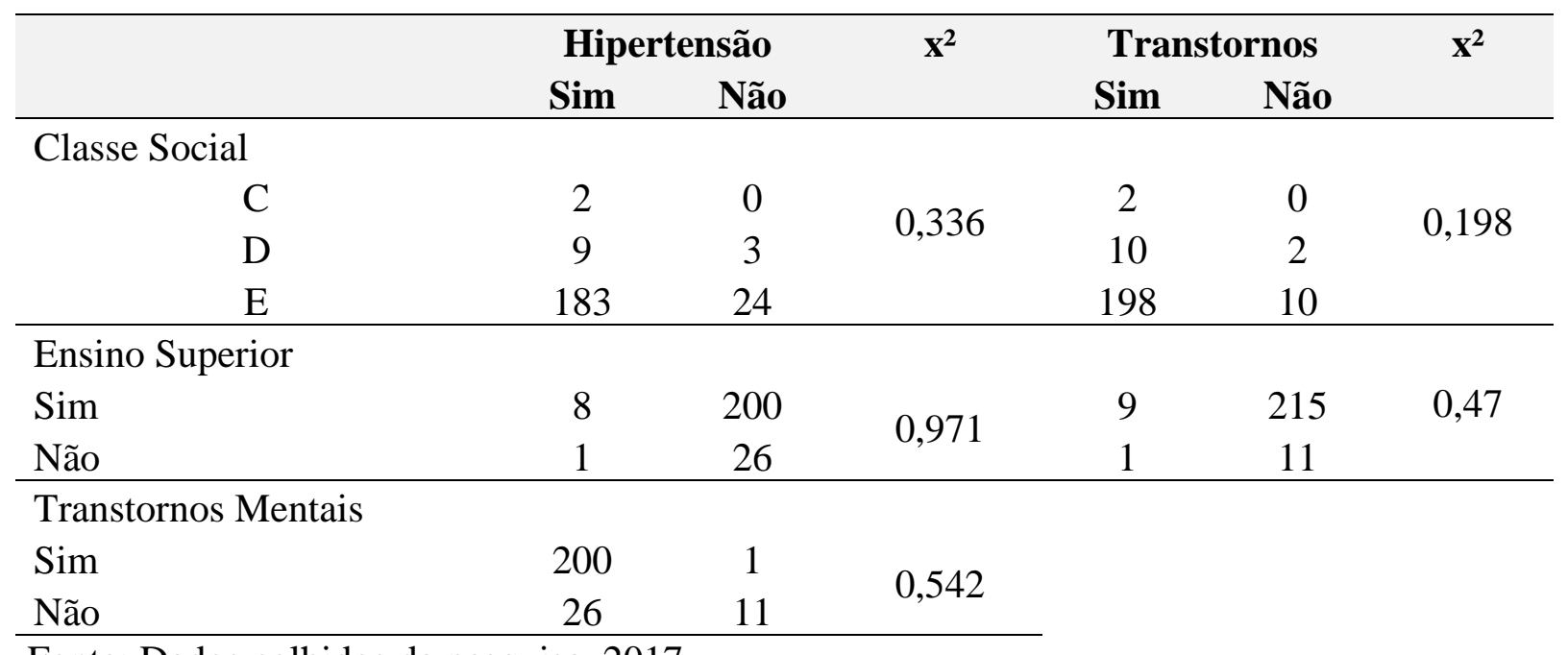

Fonte: Dados colhidos da pesquisa, 2017.

Dos 238 indivíduos analisados no quesito transtornos mentais: Quanto aos critérios classe social: 02 eram pertencentes da classe social C, 10 pertenciam à classe social D, 198 classe social E, 28 ficaram omissos no sistema. Analisando no quesito diagnostico Hipertensão Arterial, 02 eram pertencentes da classe social C, 09 eram pertencentes da classe 
social D, 183 classe social E. Quanto ao cruzamento entre os dados de transtornos mentais e hipertensão arterial: 11 não hipertensos apresentavam distúrbios psicológicos enquanto 200 apresentavam hipertensão arterial concomitantemente á distúrbios mentais.

Este presente estudos evidenciou uma relação direta entre hipertensão arterial e distúrbios psicológicos o que está em consonâncias com estudos publicados que demonstra que variáveis psicológicas exercem grandes influencia no aparecimento da Hipertensão Arterial sistêmica (FIGUEIREDO; CASTRO, 2015). Um estudo realizado com 172 idosos, $25 \%$ ( $\mathrm{p}=0,1158)$, dos idosos hipertensos demonstrou que não houve diferenças importantes entre as variáveis depressão baixas cognitivas com hipertensão, porém foi possível definir que geralmente a população com depressão e problemas cognitivos é superior á população geral (SILVA, 2014).

\section{Considerações Finais}

Os dados obtidos demonstraram uma relação significativa entre transtornos mentais e Hipertensão Arterial Sistêmica-HAS, deseja-se, que este estudo colabore positivamente nas estratégias de combate á essas condições clinicas que vitimizam milhares de indivíduos ao ano e onera o estado, tendo em expectativa que os profissionais de saúde estendam suas visões sobre a pessoa humana tendo em vista o ser de forma holística para poder exercer o cuidado com excelência e primazia. Simultaneamente deseja-se que este estudo proceda ainda auxiliar outros sobre essa concepção, instigando novas averiguações para subsidiar de táticas de enfretamento á esses problemas de saúde.

\section{Referências}

AMERICAN PSYCHIATRIC ASSOCIATION (APA). Diagnostic and statistical manual of mental disorders (DSM). 2013.

FINATTI, Betty Elmer; ALVES, Jolinda de Moraes; SILVEIRA, Ricardo de Jesus. O perfil sócioeconômico e cultural dos estudantes da Universidade Estadual de Londrina-PR. In: JORNADA INTERNACIONAL DE POLÍTICAS PÚBLICAS, 3., 2007. Anais... São Luís, MA, 2007. 
BRASIL. Ministério da Saúde. Estratégias para o cuidado da pessoa com doença crônica: hipertensão arterial sistêmica. Brasília: Ministério da Saúde, 2013. (Cadernos de Atenção Básica, n. 37).

CAVALCANTI JUNIOR, T. C.; de SOUZA, L. A. S. Relação entre o estresse e o condicionamento físico em policiais militares da Paraíba. Revista Campo do Saber, v. 2, n. 1, p. 118-136, jan./jun. 2016.

FIGUEIREDO, J. D. O.; CASTRO, E. E. C. Ajustamento criativo e estresse na hipertensão arterial sistêmica. Revista da Abordagem Gestáltica, v. 21, n. 1, p. 37-46, 2015.

GOMES-OLIVEIRA, M. H.; GORENSTEIN, C.; LOTUFO NETO, F.; ANDRADE, L. H.; WANG, Y. P. Validation of the Brazilian Portuguese version of the Beck Depression Inventory-II in a community sample. Rev Bras Psiquiatr. v. 34, n. 4, p. 389-94, 2012.

MALACHIAS, M. V. B. et al. $7^{\text {a }}$ Diretriz Brasileira de Hipertensão Arterial. Arq Bras Cardiol., v. 107, n. 3, supl. 3, p. 1-83, 2016.

MOREIRA, A. D. L. C.; GARCIA, M. C.; CAVAZZA, B. I. S. Depressão-algumas considerações. Psycologia: Saúde Mental \& Segurança Pública, v. 1, n. 1, p. 37-46, 2017.

PEREZ, Z. C. Intervenção Educativa em Adolescentes para Prevenir a Hipertensão Arterial no Adulto Jovem na UBS "Alice de Lima Barbosa"-Horizonte-CE. 2015. 31p. TCC (Especializacao em Saude da Familia) - Universidade Aberta do SUS, Fortaleza, 2015.

RABELO, R. de O.; RIBEIRO, R. G.; VASCONCELOS, L. F.; RAMYR, D.; MOREIRA, S.; OLIVEIRA, E. L. de; CARVALHO, A. M. R. de. Novas hipóteses fisiopatológicas da depressão. Mostra Científica da Farmácia, v. 2, n. 2, p. 1, 2017.

ROSSETTI, M. O. et al. O inventário de sintomas de stress para adultos de LIPP (ISSL) em servidores da polícia Federal de São Paulo. Revista Brasileira de Terapias Cognitivas, v. 4, n. 2, p. 108-119, 2008. Doi: 10.5935/1808-5687.20080018.

SILVA, P. C. D. S. da; MONTEIRO, L. A.; GRACIANO, A. D. da S.; TERRA, F. de S.; VEIGA, E. V. Avaliação da depressão em idosos com hipertensão arterial sistêmica. Northeast Network Nursing Journal, v. 15, n. 1, p. 151-157, 2014. Doi: 10.15253/2175-6783.2014000100019.

SILVA, R. M.; OLIVEIRA, D. W. D. D.; BISCARO, P. C. B.; ORTI, N. P.; SÁ-PINTO, A. C.; RAMOS-JORGE, M. L. Inquérito epidemiológico em população idosa (parte II) \&58; saúde bucal, ansiedade, depressão, estresse e uso de medicamentos \&61; Epidemiologicalsurvey in elderlypeople (II) \&58; oral health, anxiety, depression, stress, anddrugutilization. Scientia Medica, v. 26, n. 1, p. 1$8,2016$.

\section{Como citar este artigo (Formato ABNT):}

SILVA, Gilmar S.; GOMES, Valquíria S.; MACÊDO, Maria Aparecida S. S.; DUARTE, Stênio F.P. Avaliação da influência de transtornos mentais sobre a pressão arterial em idosos cadastrado em um projeto. Id on Line Revista ultidisciplinar e de Psicologia, 2018, vol.12, n.39, p.112-117. ISSN: 1981-1179.

Recebido: 15.11 .2017

Aceito: 18.11 .2017 